\title{
Cerebral arteriovenous malformation in Noonan's syndrome
}

\author{
F. Schon, J. Bowler and M. Baraitser ${ }^{1}$ \\ Department of Neurology, Atkinson Morley's Hospital, Copse Hill, London, SW20 ONE and ${ }^{1}$ Institute of \\ Neurology, National Hospital for Nervous Diseases, Queen Square, London, WC1N 3BG, UK
}

\begin{abstract}
Summary: Noonan's syndrome involves the association of multiple congenital abnormalities including neck webbing, pectus excavatum, facial anomalies with a variety of cardiac defects. In this paper the association of Noonan's syndrome with a large cerebral arteriovenous malformation is reported. Congenital cerebrovascular abnormalities are not a recognized feature of the syndrome. The paper also reviews previous reports of neurological associations with Noonan's syndrome, the commonest being mild intellectual impairment and ptosis.
\end{abstract}

\section{Introduction}

Noonan and Ehmke ${ }^{1}$ first described a syndrome of multiple congenital abnormalities in 1963 which is now thought to be a genetically determined condition with autosomal dominant inheritance. The cardinal features of the syndrome involve short stature, congenital heart disease, webbed neck, pectus excavatum and facial anomalies. ${ }^{2}$ Most review articles are agreed that the syndrome involves in addition to abnormalities of growth, cranio-facial development and cardiovascular defects, changes in the genitourinary, skeletal, ectoderm and lymphatic systems. ${ }^{3}$ However, the question of whether neurological changes occur any more often than chance is unresolved. Numerous individual case reports exist linking Noonan's syndrome (NS) with a variety of neurological disorders. In this paper a case of NS associated with a cerebral arteriovenous malformation is described and the neurological associations of NS are reviewed.

\section{Case report}

A 35 year old Caucasian male electrician presented with a 5 year history of increasing right sided ptosis, mild ptosis on the left and minor left arm weakness. His past history included bilateral congenital inguinal herniorrhaphy and surgery for left talipes equinovarus. His parents (who were nonconsanguinous) and his 5 siblings were unaffected. On examination he was of short stature $(5 \mathrm{ft} 1$ in)

Correspondence: F. Schon, Ph.D., M.R.C.P.

Accepted: 8 May 1991 with short curly black (woolly) hair. He had no eyebrows, his ears were posteriorly rotated, his neck webbed, his skin unusually lax and his chest showed pectus carinatum superiorly and pectus excavatum inferiorly (Figure 1). There was a mild scoliosis. The main neurological findings were bilateral ptosis most prominent on the right, extraocular movements in the right eye were limited with elevation, abduction and adduction being markedly impaired. There was a mild left spastic hemiparesis with brisk left-sided reflexes and bilateral extensor plantar responses. Higher mental function was normal. There was a loud cranial bruit and arterialized conjunctival vessels. Cardiac examination revealed a prominent right ventricular impulse and a pulmonary systolic murmur.

A computerized tomographic (CT) brain scan with contrast enhancement revealed an extensive basal arteriovenous malformation, with large draining veins in the region of the right cavernous sinus. Deep within the right hemisphere was a presumed porencephalic cyst (Figure 2). Cerebral angiography was not carried out. His partial third nerve palsy may well have been due to pressure from the enlarged veins in the region of the cavernous sinus.

Echocardiography showed right ventricular hypertrophy and mild tricuspid regurgitation. Doppler studies suggested slightly raised pulmonary arterial systolic pressure but arterial oxygen saturation was $97 \%$ implying any pulmonary arteriovenous shunt must be small.

The patient had no cardiovascular symptoms or signs and his chest X-ray, electrocardiograms and echocardiogram were unremarkable. 

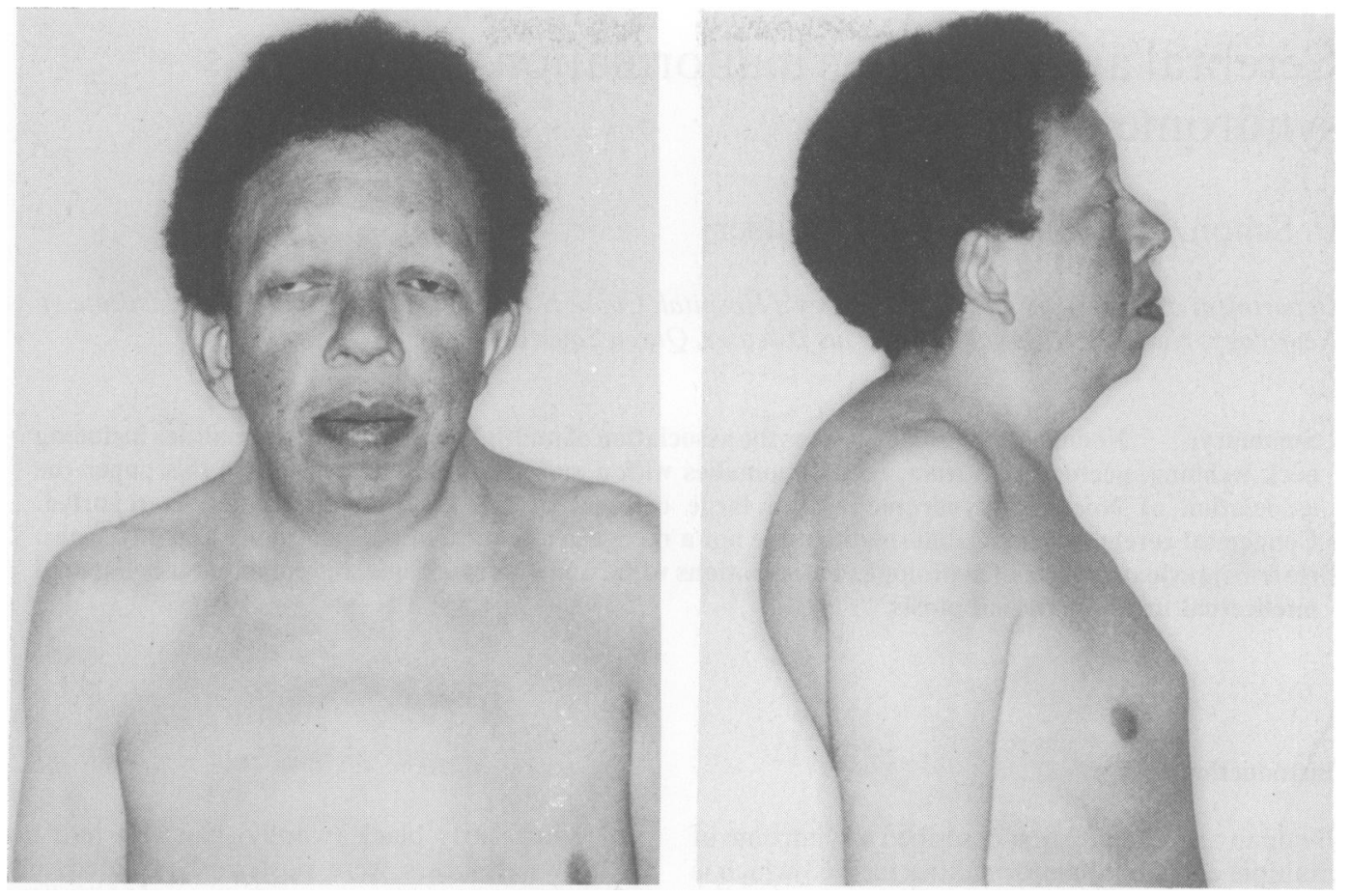

Figure 1 Patient showing tightly curled hair, absent eyebrow hair, prominent ptosis most marked on the right webbing of the neck. (Reproduced with the consent of the patient.)

\section{Discussion}

The cardiovascular abnormalities associated with NS are interesting in that they not only involve the heart itself (such as pulmonary stenosis, atrial septal defects, hypertrophic cardiomyopathy and ventricular septal defects) but also a wide variety of abnormalities of the major arteries and veins within the chest such as coarctation, patent ductus arterosis, anomalous venous drainage, and brachiocephalic vessel abnormalities. ${ }^{4.5}$ Changes in the veins and lymphatics extending beyond the thorax have also been described. ${ }^{4,5}$ It is therefore open to speculation whether cerebrovascular changes might be expected in NS. The present case involves a very extensive cerebral arteriovenous malformation. As with all isolated case reports the question arises of whether the association is purely due to chance. There are no similar cases in the literature. However, McAnena et al. ${ }^{6}$ described a case of subarachnoid haemorrhage arising from a left posterior communicating artery aneurysm in association with NS. There are no systematic studies of the cerebral vasculature in NS which would be hard to carry out during life.

A variety of other neurological conditions have been described in association with NS. The commonest is developmental delay and mild mental retardation. ${ }^{7,8}$ Ptosis is also recorded in many studies $^{9}$ without evidence of a generalized myopathy, although an association between NS and malignant hyperpyrexia has been suggested. ${ }^{10}$ Two cases of NS in association with cerebellar ectopia have been reported. ${ }^{11,12}$ It is not clear whether this is related to their short and webbed neck. A possible overlap between NS and neurofibromatosis has also been suggested in some families. ${ }^{13,14}$ There are numerous reports of NS being associated with a wide variety of isolated neurological conditions, these include a temporal lobe anomaly, ${ }^{15}$ hydrocephalus, ${ }^{2}$ cerebral abscess ${ }^{16}$ and malignant Schwannoma. ${ }^{17}$

In 1988 Reynolds et al. ${ }^{18}$ described 8 patients with multiple congenital anomalies and mental retardation which they called the cardio-facialcutaneous or CFC syndrome. These authors pointed out that there are some shared features with Noonan's syndrome. However, severe mental retardation and abnormal neurological findings are clearly much commoner in the CFC syndrome than in Noonan's syndrome.

In view of the wide range of manifestations of NS involving many systems it would perhaps be surprising if the nervous system was spared. Large studies are needed to define any involvement possibly involving computerized tomographic or magnetic resonance imaging brain screening. 

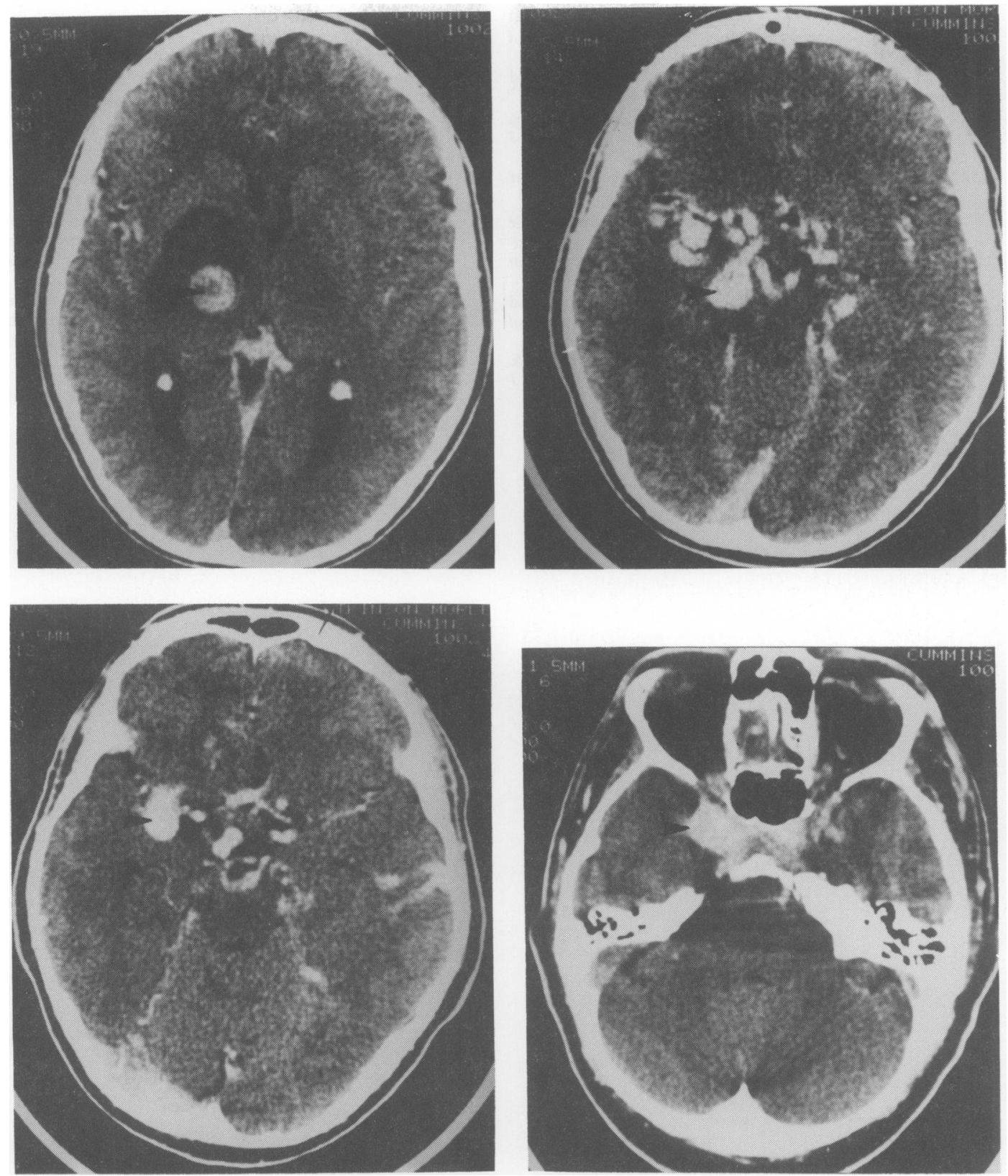

Figure 2 Computerized tomographic brain scan showing an extensive basal arteriovenous malformation with large draining veins (arrowheads) in the region of the right cavernous sinus and a cyst deep within the right hemisphere.

\section{References}

1. Noonan, J.A. \& Ehmke, D.A. Associated noncardiac malformations in children with congenital heart disease. $J$ Pediat 1983, 63: 468-470.

2. Noonan, J.A. Hypertelorism with Turner phenotype. A new syndrome with associated congenital heart disease. Am J Dis Child 1968, 116: 373-380.
3. Allanson, J.E. Noonan syndrome. J Med Genet 1987, 24: 9-13.

4. Pearl, W. Cardiovascular anomalies in Noonan's syndrome. Chest 1977, 71: 677-697.

5. Lam, J., Corno, A., Oorthuys, H.W.E. \& Marcellettic, E. Unusual combination of congenital heart lesions in a child with Noonan's syndrome. Ped Cardiol 1982, 3: 23-26. 
6. McAnena, O., Buckley, T. F. \& Padilla, J.R. Intracranial aneurysm in association with Noonan's syndrome. Irish Med $J$ 1984, 71: 140-141.

7. Money, J. \& Kalns, M.E. Noonan's syndrome: IQ and specific disabilities. Am J Dis Child 1979, 133: 846-850.

8. Nora, J.J., Nora, A.H., Sinha, A.K., Spangler, R.D. \& Lubs, H.A. Ullrich-Noonan syndrome (Turner phenotype). Am J Dis Child 1974, 127: 48-55.

9. Schwartz, D.E. Noonan's syndrome associated with ocular abnormalities. Am J Ophthalmol 1970, 73: 955.

10. Hunter, A. \& Pinsky, L. An evaluation of the possible association of malignant hyperthermia with Noonan's syndrome using serum creatinine phosphokinase levels. $J$ Pediat 1975, 96: 412-415.

11. Ball, M.J. \& Peiris, A. Chiari (type 1) malformation and syringomyelia in a patient with Noonan's syndrome. $J$ Neurol Neurosurg Psychiat 1982, 45: 753-754.

12. Kobayashil, I., Aikawa, T., Titakemiya, T., Maruyama, S. \& Takaho, K. Noonan's syndrome with syringomyelia. Jap $J$ Psych Neurol 1986, 40: 101-104.
13. Meineoke, P. Evidence that the neurofibromatosis-Noonan syndrome is a variant of Von Recklinghausen neurofibromatosis. Am J Med Genet 1987, 26: 741-745.

14. Allanson, J.E., Hall, J.G. \& Van Allen, M.I. Noonan phenotype associated with neurofibromatosis. Am J Med $\subseteq$ Genet 1985, 21: 457-462.

15. Gorke, W. Zerebrale anomalien bei Noonan syndrome. Clinik Pediatr 1980, 192: 577-581.

16. Unnithan, R.R., Bahnleyant, C.G. \& Matnewroy, V.C. Noonan syndrome. Assoc Physic India 1985, 33: 177-179.

17. Kaplan, M.S., Optiz, J.M. \& Gosset, F.R. Noonan's syndrome. A case with elevated serum alkaline phosphatase levels and malignant schwannoma of the left forearm. Am J O Dis Child 1968, 116: 359-366.

18. Reynolds, J.F., Neri, G., Jermann, J.P. et al. New multiple $\vec{\circ}$ congenital anomalies/mental retardation syndrome with cardio-facio-cutaneous involvement-The CFC syndrome. Am J Med Genet 1986, 25: 413-427. 